\title{
Cerebrospinal fluid metallomics in cerebral amyloid angiopathy: an exploratory analysis
}

\author{
Gargi Banerjee ${ }^{1,2} \circledast$. Niklas Forsgard ${ }^{3} \cdot$ Gareth Ambler $^{4} \cdot$ Ashvini Keshavan $^{5} \cdot$ Ross W. Paterson $^{5} \cdot$ Martha S. Foiani $^{6,7}$. \\ Jamie Toombs ${ }^{6,7} \cdot$ Amanda Heslegrave $^{6,7}$. Edward J. Thompson ${ }^{8}$. Michael P. Lunn ${ }^{6,8,9} \cdot$ Nick C. Fox $^{5,7}$. \\ Henrik Zetterberg ${ }^{6,7,10,11}$. Jonathan M. Schott ${ }^{5}$. David J. Werring ${ }^{1,2}$
}

Received: 28 May 2021 / Revised: 9 July 2021 / Accepted: 12 July 2021 / Published online: 22 July 2021

(c) The Author(s) 2021

\begin{abstract}
Introduction Cerebral amyloid angiopathy (CAA) is associated with symptomatic intracerebral haemorrhage. Biomarkers of clinically silent bleeding events, such as cerebrospinal fluid (CSF) ferritin and iron, might provide novel measures of disease presence and severity.

Methods We performed an exploratory study comparing CSF iron, ferritin, and other metal levels in patients with CAA, control subjects (CS) and patients with Alzheimer's disease (AD). Ferritin was measured using a latex fixation test; metal analyses were performed using inductively coupled plasma mass spectrometry.

Results CAA patients $(n=10)$ had higher levels of CSF iron than the $\mathrm{AD}(n=20)$ and CS $(n=10)$ groups (medians 23.42 , 15.48 and $17.71 \mu \mathrm{g} / \mathrm{L}$, respectively, $p=0.0015$ ); the difference between CAA and AD groups was significant in unadjusted and age-adjusted analyses. We observed a difference in CSF ferritin (medians 10.10, 7.77 and $8.01 \mathrm{ng} / \mathrm{ml}$, for CAA, AD and CS groups, respectively, $p=0.01$ ); the difference between the CAA and AD groups was significant in unadjusted, but not age-adjusted, analyses. We also observed differences between the CAA and AD groups in CSF nickel and cobalt (unadjusted analyses).

Conclusions In this exploratory study, we provide preliminary evidence for a distinct CSF metallomic profile in patients with CAA. Replication and validation of these results in larger cohorts is needed.
\end{abstract}

Keywords Alzheimer's disease $\cdot$ Cerebral amyloid angiopathy $\cdot$ Cerebrospinal fluid $\cdot$ Ferritin $\cdot$ Metallomics $\cdot$ Iron

Jonathan M. Schott and David J. Werring have contributed equally to this work.

David J. Werring

d.werring@ucl.ac.uk

1 Department of Brain Repair and Rehabilitation, Stroke Research Centre, UCL Queen Square Institute of Neurology, Russell Square House, 10-12 Russell Square, London WC1B 5EH, UK

2 National Hospital for Neurology and Neurosurgery, Queen Square, London, UK

3 Department of Clinical Chemistry, Sahlgrenska University Hospital, Gothenburg, Sweden

4 Department of Statistical Science, University College London, London, UK

5 Department of Neurodegenerative Disease, Dementia Research Centre, UCL Queen Square Institute of Neurology, London, UK
6 Department of Neurodegenerative Disease, UCL Queen Square Institute of Neurology, London, UK

7 UK Dementia Research Institute at UCL, London, UK

8 Neuroimmunology and CSF Laboratory (NICL), UCL Queen Square Institute of Neurology, London, UK

9 MRC Centre for Neuromuscular Disease, National Hospital for Neurology and Neurosurgery, London, UK

10 Clinical Neurochemistry Laboratory, Sahlgrenska University Hospital, Mölndal, Sweden

11 Department of Psychiatry and Neurochemistry, Institute of Neuroscience and Physiology, The Sahlgrenska Academy at the University of Gothenburg, Mölndal, Sweden 


\section{Introduction}

Cerebral amyloid angiopathy (CAA) is a cerebral small vessel disease characterised by amyloid-beta $(\mathrm{A} \beta)$ deposition in cortical and leptomeningeal blood vessels [4]. CAA is associated with lobar intracerebral haemorrhage; this, together with other haemorrhagic imaging markers (cerebral microbleeds, cortical superficial siderosis), forms the basis for in vivo diagnosis [8, $11,14]$. Cerebrospinal fluid (CSF) biomarkers of clinically silent bleeding events might provide a novel means of quantifying disease presence and severity; however, data in CAA are limited [13].

We performed a pilot study investigating CSF iron and ferritin levels in patients with CAA, control subjects (CS) and patients with Alzheimer's disease (AD), and how these correlated with other neurodegenerative CSF biomarkers (A $\beta-40$, A $\beta-42$, total tau, phospho-tau, and neurofilament light). We hypothesised that CSF ferritin and iron would be highest in the CAA group. In exploratory analyses, we measured concentrations of other CSF metals (nickel, chromium, zinc, manganese, cobalt and copper).

\section{Methods}

\section{Patient selection}

We included samples from the cross-sectional prospective observational BOCAA (Biomarkers and Outcomes in Cerebral Amyloid Angiopathy) study [5], and samples collected by the Specialist Cognitive Disorders Service at the National Hospital for Neurology and Neurosurgery, London, UK; group inclusion and exclusion criteria and the standardised protocol for sample collection have been provided in detail previously [3] and therefore will only be described briefly here. Patients with CAA all met "probable" modified Boston Criteria [11] and had been asymptomatic (i.e. without clinical evidence of acute intracerebral haemorrhage) in the 6 months prior to their study visits; CSF biomarkers were not used in the diagnostic process. Patients with AD had an amnestic presentation and were diagnosed on the basis of clinical assessment, imaging, and CSF measures; those with haemorrhagic imaging markers of CAA (cerebral microbleeds, cortical superficial siderosis) were excluded. Control subjects had no significant neurological diagnoses and had no evidence of significant cerebrovascular disease (including CAA) or atrophy on brain (MR) head imaging. Informed written consent was obtained for all participants.

\section{CSF analysis}

Methods for CSF processing, including measurement of $\mathrm{A} \beta-40, \mathrm{~A} \beta-42$, total tau, phospho-tau and neurofilament light, have been described previously [3].

Ferritin was measured using a latex fixation test according to manufacturer's instructions (described previously) [9]. All samples were measured on the same day by a single operator using the same reagents.

CSF metal analyses were performed by inductively coupled plasma mass spectrometry (ICP-MS) with an octopole reaction system operated in the helium collision mode (Agilent $7700 \times$ ICP-MS; Agilent Technologies, Santa Clara, Ca, USA). All samples were diluted 10 times with a basic diluent containing 1-butanol $(2 \% \mathrm{w} / \mathrm{v})$, ethylenediaminetetraacetic acid (EDTA) $(0.05 \% \mathrm{w} / \mathrm{v})$, Triton X-100 (0.05\%w/v), and ammonium hydroxide ( $1 \% \mathrm{w} / \mathrm{v})$ and were analysed in a single batch after calibration performed in the dilution medium. Germanium was used as internal standard for all elements. One quality control sample was analysed in the beginning and end of the batch (Seronorm ${ }^{\mathrm{TM}}$ Trace Elements Urine L-1, Lot no. 1011644); all element concentrations were within the stated acceptable limits.

\section{Statistics}

Statistical analysis was performed using Stata (Version 15.1). Group characteristics were compared using either one-way ANOVA (normally distributed variables), Chisquared (categorical variables) or Kruskal-Wallis (non-normally distributed data, including all biomarkers) tests. For biomarkers, if a significant difference was identified (defined as $p<0.05$ ), Dunn's test was used for post hoc comparisons, and a Bonferroni correction (resultant $p$ value multiplied by 3) applied.

In order to perform age-adjusted analyses, we used quantile regression (comparing group medians) and calculated predicted medians. We then performed post hoc pairwise comparisons of the age-adjusted medians; statistical significance was defined as Bonferroni-corrected $p<0.05$.

Spearman correlation was used to look for correlations between CSF ferritin and iron and other neurodegenerative CSF markers. Associations with a Spearman's rho value $(\rho)$ above 0.3 or below -0.3 were regarded as correlations of potential interest. 


\section{Results}

We included 10 patients with CAA, 20 patients with $\mathrm{AD}$ and 10 control subjects (Table 1). Patients with CAA were older than the two other groups, and patients with AD had lower mini-mental state examination (MMSE) scores.

In univariable comparisons (Table 1, Fig. 1), patients with CAA had higher CSF ferritin levels than the other groups (median $10.10 \mathrm{ng} / \mathrm{ml}$ in CAA group vs $7.77 \mathrm{ng} / \mathrm{ml}$ in AD group and $8.01 \mathrm{ng} / \mathrm{ml}$ in the CS group, $p=0.01$ ). Patients with CAA also had significantly higher CSF iron levels than the AD group (median $23.42 \mu \mathrm{g} / \mathrm{L}$, vs $15.48 \mu \mathrm{g} / \mathrm{L}$, $p=0.0006$ ); the difference in medians between the CAA and CS group was of a similar magnitude (median $23.42 \mu \mathrm{g} / \mathrm{L}$ vs $17.71 \mu \mathrm{g} / \mathrm{L}$ ), but did not reach statistical significance $(p=0.09)$. There were no significant differences in CSF ferritin or iron when comparing the $\mathrm{AD}$ and $\mathrm{CS}$ groups.

Table 1 Comparison of characteristics and biomarkers by group

\begin{tabular}{|c|c|c|c|c|c|c|c|}
\hline & \multirow[t]{2}{*}{ CAA $(n=10)$} & \multirow[t]{2}{*}{$\mathrm{AD}(n=20)$} & \multirow[t]{2}{*}{$\mathrm{CS}(n=10)$} & \multirow{2}{*}{$\begin{array}{l}\text { Group com- } \\
\text { parison, } p \\
\text { value }\end{array}$} & \multicolumn{3}{|c|}{$\begin{array}{l}\text { Post hoc comparisons; } p \\
\text { values }\end{array}$} \\
\hline & & & & & CAA/AD & $\mathrm{CAA} / \mathrm{CS}$ & $\mathrm{AD} / \mathrm{CS}$ \\
\hline Age, years, mean (SD) & $68.6(3.0)$ & $62.5(4.1)$ & $62.2(5.4)$ & 0.001 & - & - & - \\
\hline Sex, female, $n(\%)$ & $2(20 \%)$ & $11(55 \%)$ & $5(50 \%)$ & 0.18 & - & - & - \\
\hline MMSE, median (IQR) & $29(28$ to 30$)$ & $24(19.5$ to 26$)$ & 29 (29 to 30$)$ & $<0.001$ & - & - & - \\
\hline \multicolumn{8}{|l|}{ CSF biomarkers } \\
\hline $\begin{array}{l}\text { Ferritin, ng/ml, median } \\
\text { (IQR) }\end{array}$ & $10.10(8.37$ to 14.00$)$ & $7.77(6.39$ to 9.18$)$ & $8.01(6.94$ to 8.95$)$ & 0.0136 & 0.0060 & 0.0483 & 1.0000 \\
\hline $\begin{array}{l}\text { Iron, concentration } \\
(\mu \mathrm{g} / \mathrm{L}), \text { median }(\mathrm{IQR})\end{array}$ & $23.42(18.82$ to 25.30$)$ & $15.48(12.28$ to 18.26$)$ & $17.71(15.19$ to 20.76$)$ & 0.0015 & 0.0006 & 0.0912 & 0.2268 \\
\hline
\end{tabular}

Group comparison $p$ values were obtained using one-way ANOVA (age), Chi-squared tests (sex), or Kruskal-Wallis tests (remainder). Post hoc comparisons were made using Dunn's test; the presented $p$ values are Bonferroni-corrected

$A D$ Alzheimer's disease, $C A A$ cerebral amyloid angiopathy, $C S$ control subjects, $I Q R$ interquartile range, MMSE mini-mental state examination, $S D$ standard deviation

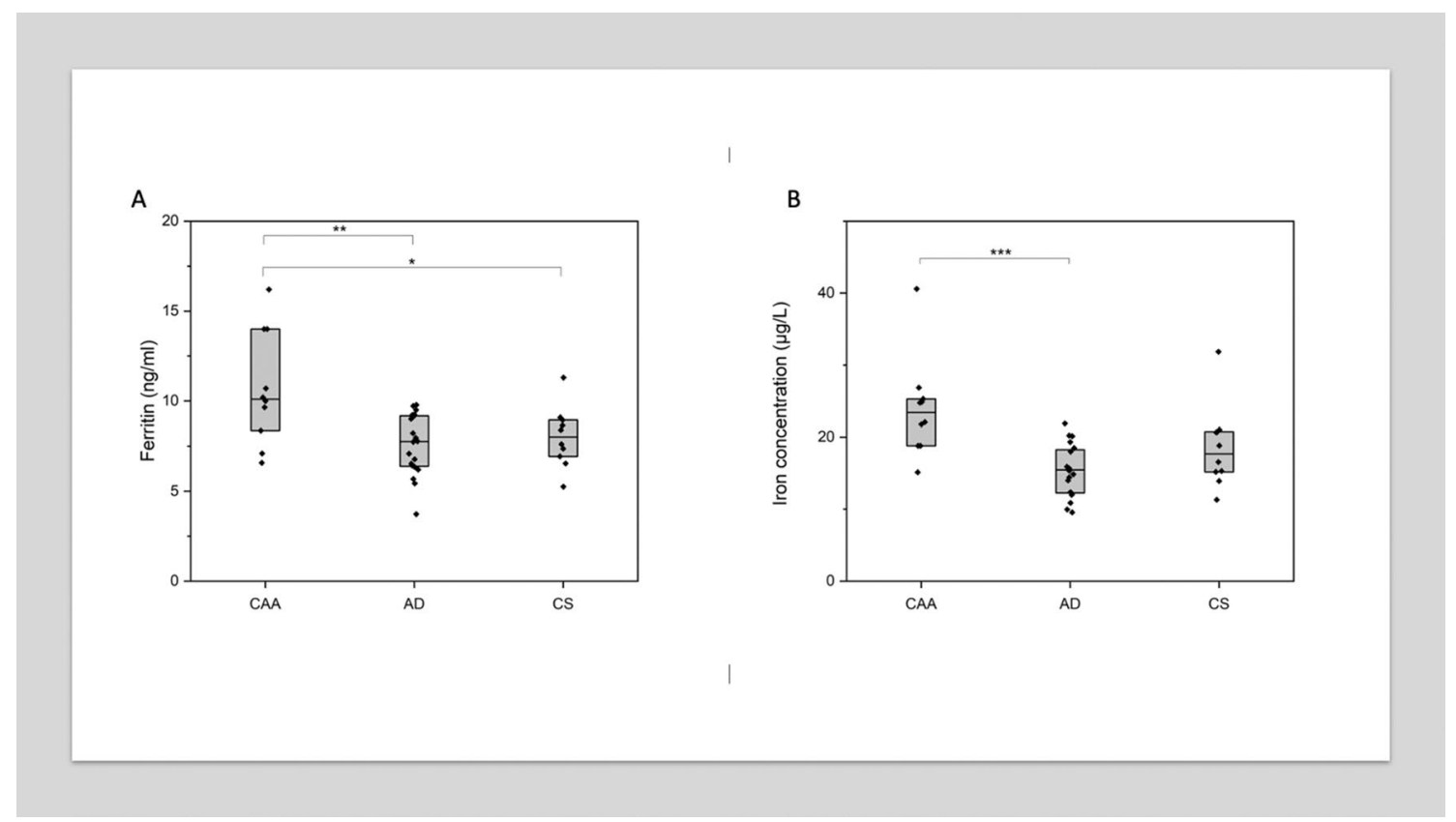

Fig. 1 CSF ferritin (A) and iron (B) by group. Horizontal line indicates median value per group; box shows 25 th and 75 th percentile. Each diamond indicates an individual data point. $p$ values are derived from post hoc Dunn's test and have been Bonferroni-corrected. *Indi- cates $p \leq 0.05$; **indicates $p \leq 0.01$; ***indicates $p \leq 0.001$. AD Alzheimer's disease, $C A A$ cerebral amyloid angiopathy, $C S$ control subjects 
In age-adjusted quantile regression (Supplementary Table 1), there was a significant difference in CSF iron between the CAA and AD groups (median difference $-7.11 \mu \mathrm{g} / \mathrm{L}$ ), but no significant difference in CSF ferritin.

We then looked for correlations between CSF ferritin and iron and other CSF biomarkers observed in neurodegenerative disease (Supplementary Table 2, Supplementary Fig. 1). When considering all groups together, there was a significant correlation between CSF A $\beta-42$ and CSF ferritin (Spearman's $\rho-0.5057, p=0.0009$ ); there was a modest correlation between CSF ferritin and A $\beta-40$ (Spearman's $\rho-0.3068, p=0.054)$, although this did not quite reach statistical significance by our definition. There was evidence of a modest correlation between CSF A $\beta-42$ and CSF iron (Spearman's $\rho-0.3122, p=0.0499$ ).

\section{Other CSF metals}

We performed further exploratory analyses for other CSF metals, the results of which are provided in the Online Supplementary Material (Supplementary Tables 3 and 4; Supplementary Fig. 2). Briefly, there were differences between the CAA and AD groups in CSF nickel (median $0.39 \mu \mathrm{g} / \mathrm{L}$ vs $0.16 \mu \mathrm{g} / \mathrm{L}, p=0.03$ ), cobalt (median $0.03 \mu \mathrm{g} / \mathrm{L}$ vs $0.01 \mu \mathrm{g} / \mathrm{L}$, $p=0.003$ ); and manganese (median $1.19 \mu \mathrm{g} / \mathrm{L}$ vs $0.84 \mu \mathrm{g} / \mathrm{L}$ ), although the latter was not quite of statistical significance $(p=0.05)$. In age-adjusted analyses, only the difference in CSF nickel remained of statistical significance (median difference $-0.25 \mu \mathrm{g} / \mathrm{L}$ ).

\section{Discussion}

In this pilot study, we provide evidence for a distinct CSF metallomic profile in patients with CAA, with higher levels of CSF iron observed in unadjusted and age-adjusted analyses. We also observed higher levels of CSF ferritin in the CAA group, reaching significance in unadjusted but not age-adjusted analyses. When considering correlations with CSF biomarkers associated with neurodegenerative disease, negative correlations of interest were observed with $A \beta-40$ (CSF ferritin), and A $\beta-42$ (CSF ferritin, CSF iron); together these measures might reflect the degree of underlying $\mathrm{A} \beta$ pathology (Supplementary Fig. 1), and a combination might provide useful diagnostic information in differentiating $\mathrm{AD}$ and CAA. In further exploratory analyses, we found differences of potential interest between the $\mathrm{AD}$ and CAA groups in CSF nickel, cobalt and manganese. CSF metallomic profile might therefore provide a novel means to differentiate between disease secondary to parenchymal versus vascular $\mathrm{A} \beta$.

We hypothesise that the elevation in CSF iron observed in CAA is due to small asymptomatic bleeding events into the subarachnoid space, which later evolve on MRI into haemorrhagic features such as cortical superficial siderosis and superficially located lobar cerebral microbleeds. In aneurysmal subarachnoid haemorrhage, iron is released into the CSF following the haemolysis of extravasated erythrocytes and subsequent degradation of haemoglobin [12]. In this context, iron is toxic, and ferritin is produced intrathecally within 24 to $48 \mathrm{~h}$ of a haemorrhage in order to sequester the free iron $[9,12]$. CSF ferritin levels in aneurysmal subarachnoid haemorrhage peak after 7 to 10 days, and can remain elevated for up to 2 months [9]. Cortical superficial siderosis in CAA develops after episodes of acute convexity subarachnoid bleeding [4], but provides no temporal information about when these haemorrhages occurred. A recent study [15] in a memory clinic population did not find any association between CSF iron and haemorrhagic MRI markers of CAA; this might reflect a lower frequency of CAA-related bleeding events (asymptomatic or symptomatic) in a memory clinic cohort than that expected in a stroke cohort, where haemorrhagic features might be more apparent. Although our observed difference in CSF ferritin was not significant in age-adjusted analyses, this might reflect a lack of power due to our small group sizes, given the biologically plausible mechanism for this observation. CSF iron and ferritin might be attractive new dynamic biomarkers for recent bleeding and thus haemorrhagic disease activity in an individual with CAA; future work is needed to explore this further.

Data from patients with AD have suggested that iron might have a role in accelerating disease progression. CSF ferritin has been associated with brain hypometabolism [7], and more rapid deteriorations in cognitive performance [2] and CSF A $\beta-42$ levels $[1,15]$. Moreover, iron is thought to enhance $A \beta$ production and pathogenicity via a number of mechanisms [10]. In our work, as in these previous reports, we observed that CSF ferritin and $A \beta-42$ were (negatively) correlated. However, we did not detect differences between controls and $\mathrm{AD}$ patients in CSF ferritin or iron; of the three groups in our study, levels of ferritin and iron were lowest in the AD group, although this was only statistically significant in comparisons (unadjusted for ferritin, unadjusted and age-adjusted for iron) with the CAA group. Our data might therefore suggest that previously described associations between CSF ferritin and poor outcomes in AD reflect the coexistence (and severity) of CAA [1, 7]. This hypothesis is supported by neuropathological data demonstrating that CAA has an independent negative effect on cognition in $\mathrm{AD}$ patients, independently of AD pathology [6]. Future studies should quantify the degree of CAA in AD patients and the impact this has on CSF iron and ferritin.

In our exploratory analyses for other CSF metals, we identified potential differences of interest between the CAA and $\mathrm{AD}$ groups in CSF nickel, manganese, and cobalt. 
Although there are some data for these and other CSF metals in $\mathrm{AD}$, to our knowledge similar findings have not been described in CAA. This might suggest that CAA results in a more widespread disruption in metal homeostasis, but the clinical and mechanistic relevance of these results are unclear; again, replication and further study in larger cohorts is needed.

We acknowledge that our exploratory study is small and therefore potentially underpowered; however, our aim was to provide hypothesis-generating data for novel biomarkers of CAA. Our preliminary data suggest that CAA might result in a distinct CSF metallomic profile; replication and validation of these results in larger cohorts is needed.

Supplementary Information The online version contains supplementary material available at https://doi.org/10.1007/s00415-021-10711-6.

Acknowledgements We would like to acknowledge our consultant colleagues from the Dementia Research Centre, who contributed patient samples, and the clinicians who performed the lumbar punctures.

Authors' contributions GB contributed to the conception and design of the work; the acquisition, analysis, and interpretation of data; drafting of the manuscript. NF contributed to the acquisition and analysis of data, and critically revised the manuscript for important intellectual content. GA contributed to the analysis and interpretation of data, and critically revised the manuscript for important intellectual content. AK and RWP contributed to the acquisition, analysis, and interpretation of data, and critically revised the manuscript for important intellectual content. MSF, JT and AH contributed to the acquisition of data, and critically revised the manuscript for important intellectual content. EJT, MPL and NCF contributed to the interpretation of data, and critically revised the manuscript for important intellectual content. HZ and JMS contributed to the acquisition and interpretation of data, and critically revised the manuscript for important intellectual content. DJW contributed to the conception and design of the work; the acquisition, analysis, and interpretation of data; and critically revised the manuscript for important intellectual content. All authors approved the version to be published and agree to be accountable for all aspects of the work in ensuring that questions related to the accuracy or integrity of any part of the work are appropriately investigated and resolved.

Funding The BOCAA study was funded by the Rosetrees Trust. GB holds a Clinical Lectureship funded by Alzheimer's Research UK (ARUK-CRF2020A-003), the Stroke Association (SA L-MP 20\100002) and the NIHR, and has previously received funding from the Rosetrees Trust. AK acknowledges the support of the Wolfson Foundation and Weston Brain Institute/Selfridges Foundation (Grant UB170045). RWP is supported by an Alzheimer's Association Clinician Scientist Fellowship, NIHR Queen Square BRC and the UK Dementia Research Institute at UCL. MPL receives support from the Department of Health's National Institute for Health Research (NIHR) Biomedical Research Centres funding scheme. HZ is a Wallenberg Scholar supported by grants from the Swedish Research Council (\#2018-02532), the European Research Council (\#681712), Swedish State Support for Clinical Research (\#ALFGBG-720931), the Alzheimer Drug Discovery Foundation (ADDF), USA (\#2018092016862), the European Union's Horizon 2020 research and innovation programme under the Marie Skłodowska-Curie grant agreement No 860197 (MIRIADE), and the UK Dementia Research Institute at UCL. JMS acknowledges the support of the National Institute for Health Research University College London Hospitals Biomedical Research
Centre (NIHR UCLH BRC), ARUK (ARUK-Network 2012-6-ICE; ARUK-PG2017-1946; ARUK-PG2017-1946), Brain Research UK (UCC14191), and Weston Brain Institute (UB170045); in addition NCF acknowledges support from the UK Dementia Research Institute as well as the NIHR UCLH BRC. DJW receives research support from the Stroke Association, the British Heart Foundation and the Rosetrees Trust. This work was undertaken at UCLH/UCL which receives a proportion of funding from the Department of Health's National Institute for Health Research (NIHR) Biomedical Research Centres funding scheme.

Data availability Additional data relating to the analyses presented in this manuscript are provided in the Supplementary Material. Analyses for the BOCAA study are ongoing; once all of these analyses are completed, the Chief Investigator (DJW) will consider applications from other researchers for access to anonymised source data.

Code availability Not applicable.

\section{Declarations}

Conflicts of interest RWP co-leads the NfL Consortium which received industry support from Abbvie, Biogen, Bristol Myers Squibb and Roche outside of this work and has given educational meeting sponsored by GE Healthcare. HZ has served at scientific advisory boards for Denali, Roche Diagnostics, Wave, Samumed, Siemens Healthineers, Pinteon Therapeutics and $\operatorname{CogRx}$, has given lectures in symposia sponsored by Fujirebio, Alzecure and Biogen, and is a co-founder of Brain Biomarker Solutions in Gothenburg AB (BBS), which is a part of the GU Ventures Incubator Program (all not related to this study). NCF has consulted for Roche; given lectures for GE and Eli Lilly; and serves on a Data Safety Monitoring Committee for Biogen (for all of which UCL received payment; all not related to this study). JMS has received research funding and PET tracer from AVID Radiopharmaceuticals (a wholly owned subsidiary of Eli Lilly); has consulted for Roche, Eli Lilly, Biogen and Merck; received royalties from Oxford University Press and Henry Stewart Talks; given education lectures sponsored by Eli Lilly, Biogen and GE; and serves on a Data Safety Monitoring Committee for Axon Neuroscience SE (not related to this study). The remaining authors have no relevant conflicts of interest to declare.

Ethical approval Ethical approval for the BOCAA study was granted in October 2015 by the NHS Health Research Authority London (REC reference 15/LO/1443). Samples collected by the Specialist Cognitive Disorders Service at the National Hospital of Neurology and Neurosurgery (NHNN), University College London Hospitals (UCLH) NHS Trust, London, UK were collected as part of a study approved by the Regional Ethics Committee at UCL.

Consent to participate Informed written consent was obtained for all participants.

Consent for publication Not applicable; this manuscript contains no identifiable data.

Open Access This article is licensed under a Creative Commons Attribution 4.0 International License, which permits use, sharing, adaptation, distribution and reproduction in any medium or format, as long as you give appropriate credit to the original author(s) and the source, provide a link to the Creative Commons licence, and indicate if changes were made. The images or other third party material in this article are included in the article's Creative Commons licence, unless indicated otherwise in a credit line to the material. If material is not included in 
the article's Creative Commons licence and your intended use is not permitted by statutory regulation or exceeds the permitted use, you will need to obtain permission directly from the copyright holder. To view a copy of this licence, visit http://creativecommons.org/licenses/by/4.0/.

\section{References}

1. Ayton S, Diouf I, Bush AI, Alzheimer's disease Neuroimaging I (2018) Evidence that iron accelerates Alzheimer's pathology: a CSF biomarker study. J Neurol Neurosurg Psychiatry 89:456-460

2. Ayton S, Faux NG, Bush AI, I Alzheimer's Disease Neuroimaging (2015) Ferritin levels in the cerebrospinal fluid predict Alzheimer's disease outcomes and are regulated by APOE. Nat Commun 6:6760

3. Banerjee G, Ambler G, Keshavan A, Paterson RW, Foiani MS, Toombs J, Heslegrave A, Dickson JC, Fraioli F, Groves AM, Lunn MP, Fox NC, Zetterberg H, Schott JM, Werring DJ (2020) Cerebrospinal fluid biomarkers in cerebral amyloid angiopathy. J Alzheimer's Dis JAD 74:1189-1201

4. Banerjee G, Carare R, Cordonnier C, Greenberg SM, Schneider JA, Smith EE, Buchem MV, Grond JV, Verbeek MM, Werring DJ (2017) The increasing impact of cerebral amyloid angiopathy: essential new insights for clinical practice. J Neurol Neurosurg Psychiatry 88:982-994

5. Banerjee G, Werring DJ (2020) Feasibility of clinical trial recruitment for cerebral amyloid angiopathy: a specialist single centre experience. J Neurol Sci 409:116580

6. Boyle PA, Yu L, Nag S, Leurgans S, Wilson RS, Bennett DA, Schneider JA (2015) Cerebral amyloid angiopathy and cognitive outcomes in community-based older persons. Neurology 85:1930-1936

7. Diouf I, Fazlollahi A, Bush AI, Ayton S, Alzheimer's disease Neuroimaging I (2019) Cerebrospinal fluid ferritin levels predict brain hypometabolism in people with underlying beta-amyloid pathology. Neurobiol Dis 124:335-339

8. Greenberg SM, Al-Shahi Salman R, Biessels GJ, van Buchem M, Cordonnier C, Lee JM, Montaner J, Schneider JA, Smith EE, Vernooij M, Werring DJ (2014) Outcome markers for clinical trials in cerebral amyloid angiopathy. Lancet Neurol 13:419-428

9. Keir G, Tasdemir N, Thompson EJ (1993) Cerebrospinal fluid ferritin in brain necrosis: evidence for local synthesis. Clin Chim Acta 216:153-166

10. Lane DJR, Ayton S, Bush AI (2018) Iron and Alzheimer's disease: an update on emerging mechanisms. J Alzheimer's Dis JAD 64:S379-S395

11. Linn J, Halpin A, Demaerel P, Ruhland J, Giese AD, Dichgans M, van Buchem MA, Bruckmann H, Greenberg SM (2010) Prevalence of superficial siderosis in patients with cerebral amyloid angiopathy. Neurology 74:1346-1350

12. Petzold A, Worthington V, Appleby I, Kerr ME, Kitchen N, Smith M (2011) Cerebrospinal fluid ferritin level, a sensitive diagnostic test in late-presenting subarachnoid hemorrhage. J Stroke Cerebrovasc Dis 20:489-493

13. Renard D, Thouvenot E (2017) CSF RBC count in successful first-attempt lumbar puncture: the interest of atraumatic needle use. Neurol Sci 38:2189-2193

14. Rodrigues MA, Samarasekera N, Lerpiniere C, Humphreys C, McCarron MO, White PM, Nicoll JAR, Sudlow CLM, Cordonnier C, Wardlaw JM, Smith C, Al-Shahi Salman R (2018) The Edinburgh CT and genetic diagnostic criteria for lobar intracerebral haemorrhage associated with cerebral amyloid angiopathy: model development and diagnostic test accuracy study. Lancet Neurol 17:232-240

15. Shams M, Martola J, Charidimou A, Granberg T, Ferreira D, Westman E, Wintermark M, Iv M, Larvie M, Kristoffersen Wiberg M, Kaijser M, Forsgard N, Zetterberg H, Wahlund LO, Shams S (2020) Cerebrospinal fluid metals and the association with cerebral small vessel disease. J Alzheimer's Dis JAD 78:1229-1236 